\title{
Photometric scaling relations for bulges of galaxies
}

\author{
C. D. Ravikumar ${ }^{1}$, S. Barway ${ }^{2}$, A. Kembhavi ${ }^{3}$, B. Mobasher ${ }^{4}$, and V. C. Kuriakose ${ }^{5}$
}

1 GEPI, Observatoire de Paris-Meudon, 92195 Meudon, France

e-mail: Chazhiyat. Ravikumar@obspm.fr

2 School of Studies in Physics, Pt. Ravishankar Shukla University, Raipur, India e-mail: sudhan@iucaa.ernet.in

3 Inter University Centre for Astronomy and Astrophysics, Post Bag 4, Ganeshkhind, Pune 411007, India e-mail: akk@iucaa.ernet.in

${ }^{4}$ Space Telescope Science Institute, 3700 San Martin Drive, Baltimore MD 21218, USA e-mail: mobasher@stsci.edu

5 Department of Physics, Cochin University of Science and Technology, Kochi 682022, India e-mail: vck@cusat.ac.in

Received 1 June 2005 / Accepted 7 September 2005

\section{ABSTRACT}

We describe the photometric parameters of the bulges of galaxies of different Hubble types including ellipticals, lenticulars, early and late type spirals and early type dwarf galaxies. Analyzing the distributions of various photometric parameters and two- and three-dimensional correlations between them, we find that there is a difference in the correlations exhibited by bright $\left(M_{\mathrm{K}}<-22\right)$ and faint bulges, irrespective of their Hubble type. Importantly, the bright bulges, which include typically E/S0 galaxies and bulges of early type spirals, are tightly distributed around a common photometric plane (PP), while their fainter counterparts, mainly bulges of late type spirals and dwarf galaxies show significant deviation from the planar distribution. We show that the specific entropy, determined from the bulge structural parameters, systematically increases as we move from late to early Hubble types. We interpret this as evidence for hierarchical merging and passive evolution scenarios for bright and faint bulges respectively.

Key words. galaxies: clusters: general - galaxies: bulges - galaxies: fundamental parameters - Galaxy: structure

\section{Introduction}

Galaxies show a wide variety of morphologies. Since the work of Hubble (1936) attempts have been made to classify these morphologies according to their apparent shape, structure, elongation etc. The wide ranges seen in their properties such as luminosity and color, and in parameters defining their large scale structure, indicate that galaxies are formed through different mechanisms and are in a state of constant evolution. A detailed analysis of the distribution of such properties, and the correlations between them, helps in understanding the systematics involved in the structure, formation and evolution of galaxies.

The ratio of bulge to disk luminosity in galaxies decreases along the Hubble sequence. Elliptical galaxies (Es), which are wholly dominated by their bulges, show considerable homogeneity, mainly characterized by the existence of the fundamental plane (FP), a tight correlation between central velocity dispersion $(\sigma)$ - effective radius $\left(r_{\mathrm{e}}\right)$ - effective mean surface brightness $\left(\left\langle\mu_{\mathrm{b}}\left(<r_{\mathrm{e}}\right)\right\rangle\right)-$ (e.g., Dressler et al. 1987; Djorgovski \& Davis 1987) with little intrinsic scatter. The homogeneity extends to many other correlations as well, like the color-magnitude (CM, Bower et al. 1992; Andreon 2003), the Faber-Jackson (1976) and Kormendy (1985) relations. Lenticulars (SOs) show striking similarities with Es in their abundance near cluster centers. The properties of the bulges of lenticulars follow closely the relations obeyed by Es, while their disks show structural similarities with the disks of spiral galaxies (Mathieu et al. 2002), sometimes with larger scatter (Hinz et al. 2003) towards their faint end. Spiral galaxies have more complex structures, with the disk having spiral arms whose characteristic too change along the Hubble sequence. In this paper, we study the photometric properties of the bulges of different Hubble types for galaxies.

A systematic and quantitative understanding of the morphological structure in galaxies is obtained by analyzing their surface brightness (SB) distribution to separate their bulge and disk properties. In the simplest approach, this is achieved by parameterizing the radial structure of the SB profile in terms of empirical functions with a small number of free parameters. The SB profile of discs is satisfactorily fitted by an 
exponential function characterized by a central surface brightness and a disk scale length (Freeman 1970; also see Grosbøl 1985; Courteau 1996). The situation with bulges is somewhat more complicated. The bulge dominated surface brightness profiles of ellipticals were found to be reasonably well fitted by the two parameter de Vaucouleurs' $r^{1 / 4}$ law (de Vaucouleurs 1959). However, in recent years better quality CCD data have shown that the generalization of $r^{1 / 4}$ to a $r^{1 / n}$ law, first proposed by Sérsic (1968), represents the SB profiles better. The $r^{1 / n}$ law also provides satisfactory fits to the bulges of lenticular and spiral galaxies. The significant correlations that the Sérsic index $n$ shares with other photometric parameters like luminosity and central surface brightness (Young \& Currie 1994; Andredakis et al. 1995; Binggeli \& Jerjen 1998; Khosroshahi et al. 2000a,b; Trujillo et al. 2001; Trujillo et al. 2002; Gerbal et al. 1997; Lima-Neto et al. 1999; Márquez et al. 2000, 2001), with spectroscopic parameters like central velocity dispersion $\sigma$ (e.g. Graham \& Colless 1997) and $[\mathrm{Mg} / \mathrm{Fe}]$ abundance ratios (Vazdekis et al. 2004) and also with the mass of the galaxy's (central) super-massive black hole (Graham et al. 2001) suggest that this generalization provides important information regarding the physical processes involved in the evolution of the bulges.

An attempt to understand and interpret the observed correlations from a theoretical point of view has been made by Gerbal et al. (1997), Lima-Neto et al. (1999), and Márquez et al. (2001). These authors consider elliptical galaxies to be in a state of quasi-equilibrium characterized by a local maximum in the specific entropy of the system. Studies of simulations of successive mergers of elliptical galaxies showed a systematic increase in the specific entropy with the merging history (Márquez et al. 2000), suggesting that the specific entropy can be used as a potential tool in understanding the evolution of galaxies.

In this paper, we will consider the quantitative decomposition of galaxies of different Hubble types into the bulge and disk components, and consider in detail various relations between the bulge properties. We will defer the discussion of disk properties to a later work. The paper is arranged as follows. In Sect. 2 we introduce the sample used in this analysis. Section 3 deals with the determination of the structural parameters of early type galaxies in $K$ band for two rich clusters Abell 2199 and Abell 2634 using the full two dimensional bulge-disc decomposition algorithm, fitgal. We describe the various photometric properties in Sect. 4 and show that the brighter $\left(M_{\mathrm{K}}<-22\right)$ and fainter bulges, irrespective of their Hubble type, fill distinct regions in their parametric correlations. Assuming gravo-thermal properties to the bulges, we then estimate their specific entropies in Sect. 5 and attempt to provide a robust evolution scenario of galaxies. In Sect. 6 we conclude with a discussion of our results and their implications.

\section{The sample}

We study the bulges of galaxies of different types. For this purpose we have compiled samples of cluster ellipticals, lenticulars, early and late type spirals, and early type dwarf galaxies. All the galaxies in our sample, except the dwarf galaxies, were observed in the near infra red, the advantages of this band being its sensitivity to the old population of stars and the capability to penetrate the dust. A brief description of the sample is given below.

- A set of 34 elliptical galaxies in two nearby clusters, Abell 2199 (20 galaxies) and A2634 (14 galaxies) at redshift $z=0.031$ and 0.030 respectively. The sample contains elliptical galaxies that are spectroscopically confirmed members of these clusters with reliable velocity dispersion and optical photometry from a study by Lucey et al. (1997). These galaxies were chosen to have optical half-light radius less than 1 arcmin, to fit in the field of view of the Infra-red Array Detector (IRCAM3) on the United Kingdom Infrared Observatory (UKIRT). The bulge disk decomposition algorithm we used for determining the photometric structural parameters requires that the surface profile be fitted by a smooth bulge + disk model. Hence galaxies with complicated morphologies, like strongly interacting galaxies, were removed from the original sample. However such galaxies constitute only a small fraction of the sample, and hence their removal did not affect the statistical completeness of the sample. This final criterion is applicable to all the following samples.

- A set of 42 ellipticals from the Coma cluster analyzed by Khosroshahi et al. (2000b). These galaxies were taken from a previous study (Mobasher et al. 1999) which originally contained 48 ellipticals applying the same selection criteria for our Abell cluster ellipticals from the spectroscopic and optical photometric catalogue by Lucey et al. (1991).

- A sample of 37 confirmed field lenticular galaxies observed in the $K^{\prime}$ band from the Observatorio Astronomico Nacional $2.1 \mathrm{~m}$ telescope at San Pedro Martir, Mexico, by Barway et al. (2005). These galaxies from the Uppsala General Catalogue (UGC) have $B<14$, angular diameter $D_{25}<3$ arcmin and declination $5^{\circ}<\delta<64^{\circ}$. The galaxies in our sample were chosen in an unbiased fashion from the larger complete sample with the abovementioned selection criteria, after removing possible mis-classifications by analyzing their $K$ band images.

- A set of 26 bulges of mostly early-type spirals from a magnitude and size limited complete sample from the UGC constructed by Balcells \& Peletier (1994) from observations in the $K$ band. The sample is complete with right ascension between $13^{\mathrm{h}}$ and $24^{\mathrm{h}}$, declination above $-2^{\circ}$, spirals earlier than Sc, $B<14$, major axis diameter larger than 2 arcmin, absolute galactic latitude larger than $20^{\circ}$ and axis ratio in $B$ larger than 1.56. The final criterion makes the sample biased towards being highly inclined (inclination angle greater than $50^{\circ}$ ). Further, the smoothness of the surface profile was guaranteed by avoiding galaxies that are irregular, strongly interacting, barred or with very strong dust lines up to the center of the galaxy. We have taken the bulge and disk parameters for this sample from the study by Khosroshahi et al. (2000a), who used the same programs and techniques as we do.

- Bulges of 40 bright $\left(B_{\mathrm{T}}<12\right)$ spiral galaxies, mostly late-type, observed in $K$ band and analyzed by 
Möllenhoff \& Heidt (2001). The sample consists of galaxies with $B<12$ of Hubble type Sa to Sc, without strong bars, selected from the Revised Shapley Ames Catalog (Sandage \& Tammann 1981).

- Early type dwarf galaxies from a study by Binggeli \& Jerjen (1998), which contains 128 highly resolved dE and dS0 profiles that are well described by the Sérsic law. These galaxies were selected from the $B$ band photometry of a complete sample with $B<18$ by Binggeli \& Cameron (1993). We have used the whole sample to represent early type dwarfs, as the dEs and dSOs do not show any distinguishable differences in their photometric correlations as shown in Binggeli \& Jerjen (1998), Also, we have converted the $B$ band photometry to that in the $K$ band assuming an average color $B-K=3.2$. Given the fact that most smooth dwarf galaxies contain a homogeneous single component population, the application of constant color is justifiable. The main motivation to include the dwarf galaxies in this study is to address their properties in relation to their counter parts amongst other Hubble types.

Further details on the selection and data reduction can be had from the references mentioned above. We assume that the Hubble constant and the cosmological deceleration parameter are given by $H_{0}=50 \mathrm{~km} \mathrm{~s}^{-1} \mathrm{Mpc}^{-1}$ and $q_{0}=0.5$ respectively.

\section{Morphological parameters}

Galaxies classified as elliptical in the classical catalogues generally have smooth elliptical isophotes, with little evidence of morphological peculiarities, dust etc. seen in survey plates. Later observations of such galaxies with CCD detectors have revealed a host of features, but these typically are faint enough to still make it possible to use simple laws to describe the large scale light distribution. The most widely used relation to describe the surface brightness distribution in elliptical galaxies at optical and near infra-red wavelengths, $I_{\mathrm{b}}(r)$, is the de Vaucouleurs law. This law, with its two free parameters, the central surface brightness $I_{\mathrm{b}}(0)$ and the half-light radius $r_{\mathrm{e}}$ (defined as the radius of the central region of the galaxy containing half its total light), provides a surprisingly good approximation to the observed surface brightness distribution of many elliptical galaxies. But with high sensitivity CCD observations becoming available, deviations from the simple $r^{1 / 4}$ law have been noticed, and a three parameter generalization, first proposed by Sérsic (1968), has been widely used (see Khosroshahi et al. 2000a, for a discussion and references). The Sérsic law is given by

$I_{\mathrm{b}}(r)=I_{\mathrm{b}}(0) \mathrm{e}^{-2.303 b_{n}\left(r / r_{\mathrm{e}}\right)^{1 / n}}$,

where $n$ is a parameter to be determined from observations for each galaxy. The constant $b_{n}$, which depends on $n$, is chosen so that $r_{\mathrm{e}}$ remains the half-light radius. For a given $n, b_{n}$ can be obtained as a root of the equation

$P\left(2 n, 2.303 b_{n}\right)=0.5$,

where $P(a, x)$ is the incomplete Gamma function (see e.g. Press et al. 1992).
The surface brightness profile of the disk is well approximated to be an exponential (Freeman 1970),

$I_{\mathrm{d}}(r)=I_{\mathrm{d}}(0) \mathrm{e}^{\left(-r / r_{\mathrm{d}}\right)}$,

where $I_{\mathrm{d}}(0)$ and $r_{\mathrm{d}}$ are the central disk intensity and the disk scale radius respectively. For extracting the bulge and disk parameters of the Abell cluster ellipticals, we have used the algorithm fitgal, a full two dimensional routine, involving $\chi_{v}^{2}$ minimization to determine the quality of the fit obtained. This algorithm is described in Wadadekar et al. (1999). For the Coma ellipticals and bulges of early type spirals in our sample, the photometric parameters have been obtained using the program fitgal by Khosroshahi et al. (2000b,a, respectively). For the lenticulars, the image analysis has been described by Barway et al. (2005), and the photometric parameters have been obtained again using fitgal (Barway et al. to be submitted). For the late type bulges and the dwarf galaxies we use results from Möllenhoff \& Heidt (2001) and Binggeli \& Cameron (1993) respectively. Even though different procedures for extracting structural parameters can introduce some systematics in the derived parameters, we anticipate the effect to be negligible, considering the fact that all the sample galaxies were selected on the basis of the smoothness in their surface brightness profiles. We would like to note here that the definition of morphological parameters used in some of the papers cited above can be different. In such cases we have made the necessary transformations to convert all parameters to the form used by us.

\section{Distributions and correlations}

We consider in this section the distribution of various parameters for different galaxy types and correlations between the parameters. Any observed correlations can provide constraints on galaxy formation and evolution models. Also, as shown by the example of the FP and the Kormendy relation (Kormendy 1977) and the relationship between $n$ and bulge scale radius (Young \& Currie 1994; Lima Neto et al. 1999), the observed correlations with small scatter can provide useful distance indicators to galaxies.

\subsection{Distributions}

In Fig. 1 we show the histogram of Sérsic bulge parameters, $n$, $\log r_{\mathrm{e}}$, and $\mu_{\mathrm{b}}(0)$, for the different sets of galaxies in our sample. Clearly it can be seen that dwarf galaxies (dEs) form a different distribution compared to all other types. The Sérsic index for dEs has a conspicuous peak at $n \sim 1$, and there are very few galaxies with $n \geq 2$. For the ellipticals $n$ peaks around $n \sim 4$, with a broad spread to lower values (this is the reason why de Vaucouleurs' law with $n=4$ provides a reasonable fit to many Es). As we move along the Hubble sequence, the average $n$ values becomes smaller, but the spread remains large with no evident peak. The distribution of $\mu_{\mathrm{b}}(0)$ for bulges of early type spirals show a close resemblance with Es and bright S0s, while the bulges of late type spirals overlap with the bright end of dwarf galaxies and faint end of other early type bulges. The effective radius decreases on average along the Hubble sequence. 


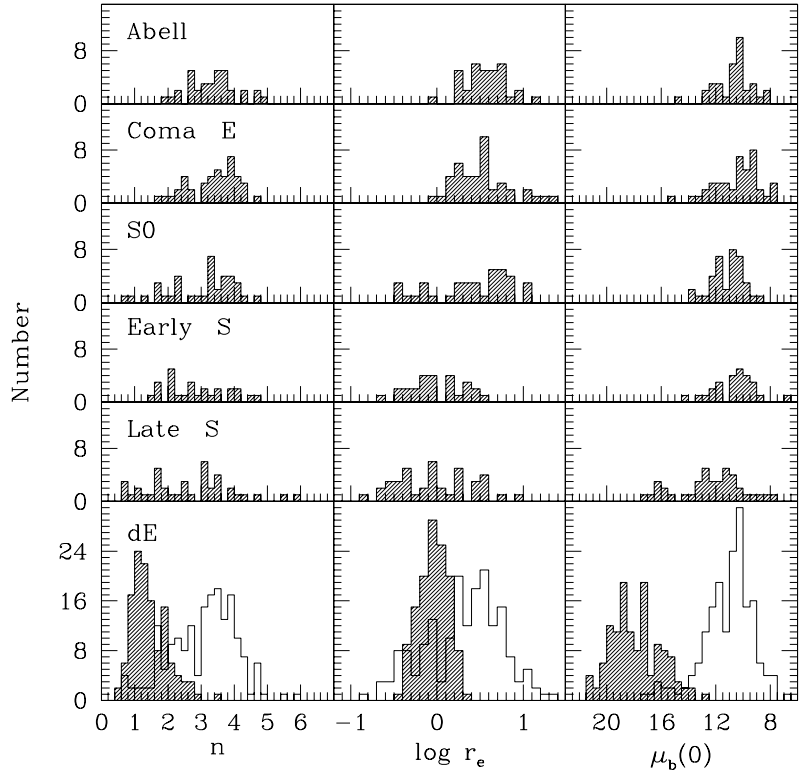

Fig. 1. Distribution of Sérsic index $(n)$, half-light radius $\left(\log r_{\mathrm{e}}\right.$ in $\left.\mathrm{kpc}\right)$ and unconvolved central surface brightness $\left(\mu_{\mathrm{b}}(0)\right.$ in mag $\left.\operatorname{arcsec}^{-2}\right)$. The bottom panel shows histograms for dwarf ellipticals (shaded) and the rest of the sample taken together (open).

However, contrary to the $\mu_{\mathrm{b}}(0)$ distribution where the dwarfs have a different distribution from the other types, in the case of $r_{\mathrm{e}}$, there is a significant overlap of the dEs with the other galaxies. Some bulges of S0s and spirals show smaller $r_{\mathrm{e}}$ than a majority of dEs. These distributions show that there is no apparent difference between the Abell and Coma cluster ellipticals in our sample. Application of the Kolmogorov-Smirnov test confirms that distributions for the two sets of cluster ellipticals are not significantly different, and therefore in our subsequent discussions we will be treating all the cluster ellipticals in our sample as a single group.

The effect of the distribution of the three basic parameters shown in Fig. 1, on the bulge luminosity is such that it decreases systematically along the Hubble sequence. In Fig. 2 we show the histogram for absolute magnitude for the different types. We have shown in Fig. 3 the distribution of the three basic parameters and the luminosity for all the bulges in our sample taken together. It clearly shows the division of the whole sample into two distinct groups. However, there is a significant overlap of all the bulge parameters and galaxy types in the two groups. This kind of a dichotomy in the structural parameters and their correlations has been reported before in the literature. Kormendy (1985) observed from his study on "ellipsoidal stellar systems" that bright Es show remarkable discontinuity in their parameter correlations from those for dwarf spheroidals (see also Capaccioli et al. 1992). This has led Kormendy \& Bender (1996) to suggest a modifications to the Hubble classification based on the apparent elongation and its association with rotation. The dichotomy between the properties of bulges is not limited to ellipticals. van den Bergh (1994), analyzing a sample of S0s, came to the conclusion that faint S0s $\left(M_{\mathrm{B}}>-19.5\right)$ are statistically more likely to be prolate than their brighter counterparts. Recent studies suggest that the bulges of early

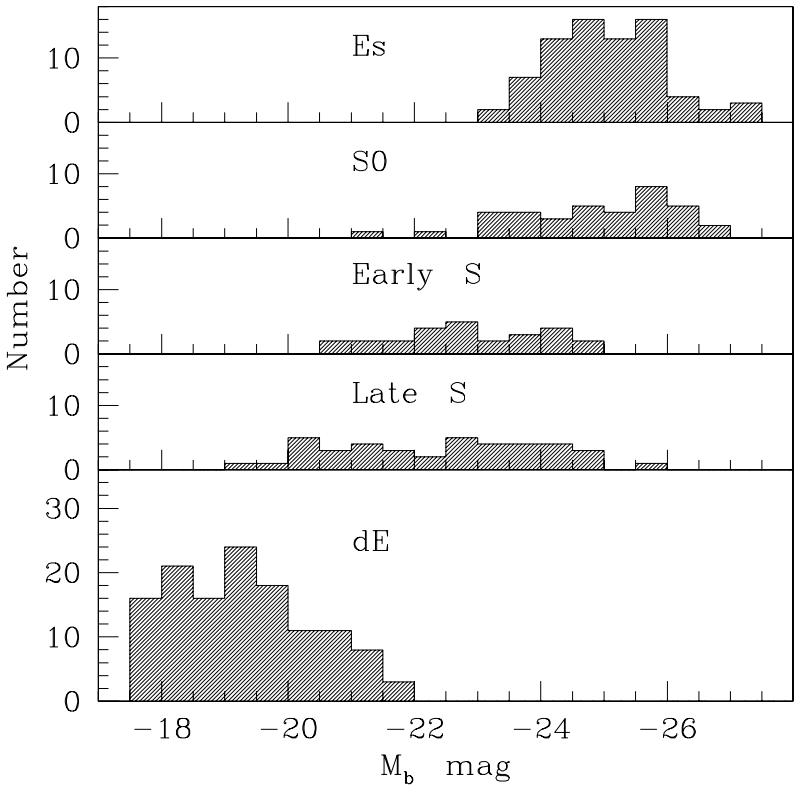

Fig. 2. Histogram of absolute $(K)$ magnitudes for bulges. There is a systematic increase in the luminosity towards early type galaxies suggesting the dominance of bulges there.
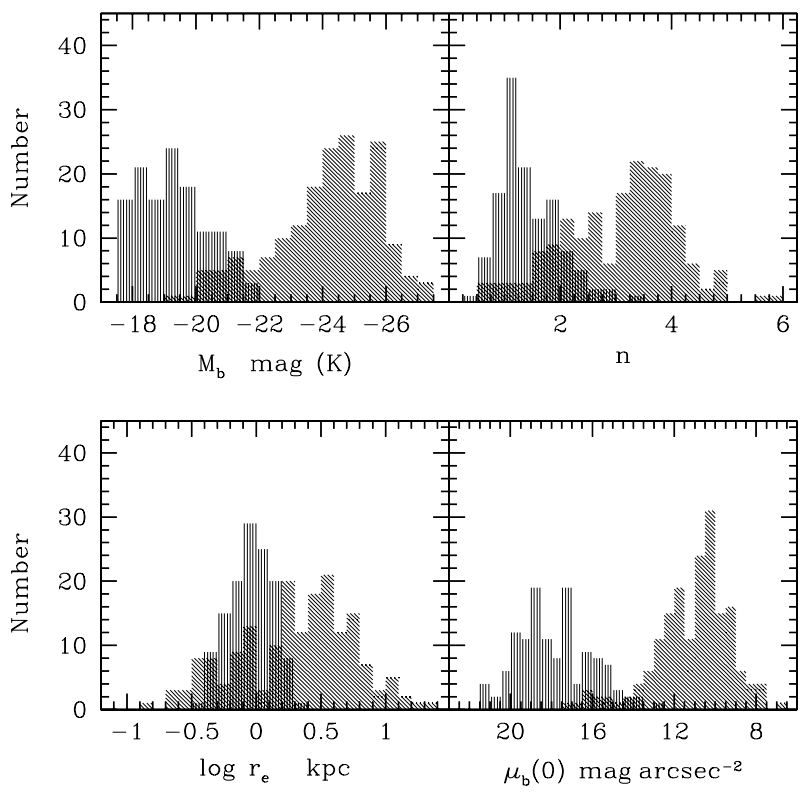

Fig. 3. Combined histogram for the structural properties of bulges in our sample. There seems to exist two distinct groups in their structural properties, irrespective of the Hubble type they belongs to.

and late type spirals seems to follow distinct distributions in their elongation (Fathi \& Peletier 2003) as well as in luminosities (the luminosity distribution is of course affected by the role that bulges play in the Hubble classification scheme). The dichotomy shown in Fig. 3 does not seem to result from the uneven sample size for the different Hubble types, since there are significant differences in the population of galaxies in the two groups for different parameters. The important observation we make here is that the dichotomy does not depend on the Hubble type, it rather seems to be a more fundamental property of the 


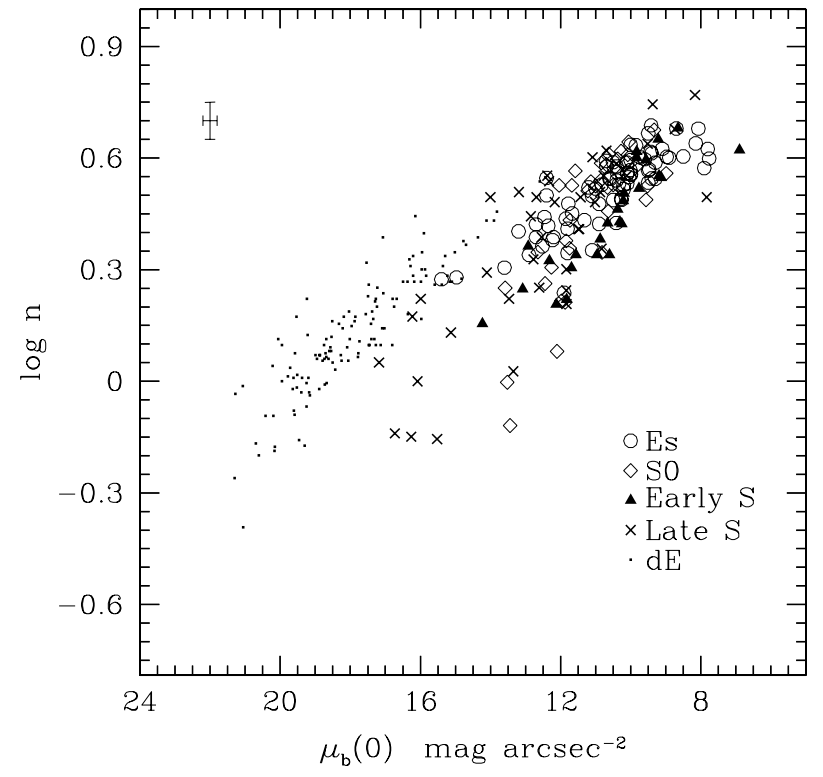

Fig. 4. Logarithm of the Sérsic index $n$ as a function of unconvolved bulge central surface brightness.

bulges that might be closely related to their formation and evolution processes.

\subsection{Correlations}

The three basic parameters involved in the Sérsic law given in Eq. (1), $I_{\mathrm{b}}(0), r_{\mathrm{e}}$ and $n$, specify the surface brightness profile completely. $I_{\mathrm{b}}(0)$ determines how bright the galaxy appears, $r_{\mathrm{e}}$ decides the extent of the galaxy and $n$ controls the "rate" of the fall of intensity within the galaxy; the larger the value of $n$, the quicker is the fall in the intensity within the half light radius. So for large values of $n$, it is necessary to have a bright core and a reasonably large $r_{\mathrm{e}}$ for the galaxy to be "visible" in the band. Hence positive correlations (with some scatter) of $n$ with both $I_{\mathrm{b}}(0)$ and $r_{\mathrm{e}}$ are expected, assuming galaxies obey the Sérsic law over the entire galaxy (e.g. D'Onofrio et al. 1994; Khosroshahi et al. 2000b; Trujillo et al. 2002).

In Fig. 4 we show a plot between $\mu_{\mathrm{b}}(0)$ and $n$ for the entire sample. In the case of the cluster ellipticals, there is a good linear correlation (correlation coefficient $=-0.90$, significance greater than $99.99 \%$, rms scatter around the best fit line measured along $\log n$ axis $=0.26$ ), The dwarf ellipticals too show a good correlation, but there is pronounced departure from linearity at the lower values of $n$. The dwarf galaxies seem to lie on the extrapolation of the trend in ellipticals to lower $n$ values, but this is to be treated with some caution, since we have used an average color to transform the observed dwarf galaxy magnitudes in the $B$ band to the $K$ band. As for the bulges of spirals, early type bulges again show a good correlation $(-0.88$, $>99.99 \%$ ) and merge with ellipticals (see also Khosroshahi et al. 2000b), while late type bulges show a large scatter and departure from linearity. The bulges of brighter lenticulars mix with the ellipticals, while their fainter counterparts show large scatter and curvature.

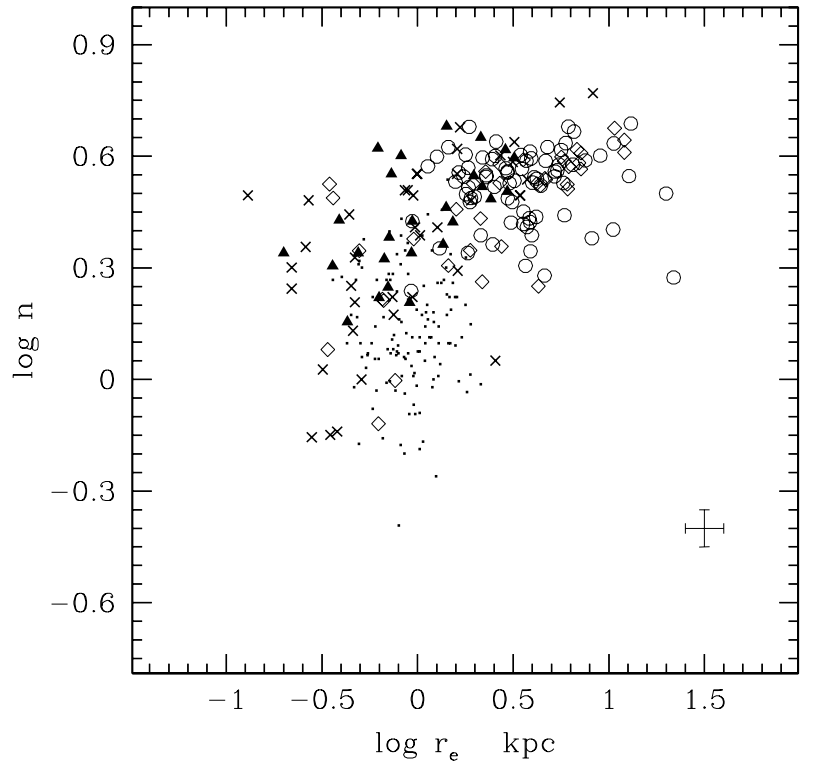

Fig. 5. Sérsic index vs. effective radius. The symbols are as used in Fig. 4. Typical error bars are also shown at the lower right corner.

A plot of the Sérsic index against the effective radius (Fig. 5) shows the presence of two broad distributions, but now without a good correlation within each group. It is seen from the figure that Es and brighter bulges of other Hubble types form a group, while the fainter (and smaller) bulges of S0s and spirals form another group similar to that of dEs.

\subsection{Kormendy relation}

Kormendy (1977) noticed that for a sample of large elliptical galaxies, the central surface brightness $\mu_{\mathrm{b}}(0)$ was correlated with $\log r_{\mathrm{e}}$. The two parameters here were determined by fitting de Vaucouleurs' law to the SB distribution. Operationally, it has been found in such investigations that it is convenient to use the mean surface brightness within $r_{\mathrm{e}},\left\langle\mu_{\mathrm{b}}\left(<r_{\mathrm{e}}\right)\right\rangle$ rather than $\mu_{\mathrm{b}}(0)$ in plotting correlations like the Kormendy relation and the fundamental plane, as the former can be more robustly determined from observation data, and the two differ from each other by a constant when de Vaucouleurs' law is used: $\left\langle\mu_{\mathrm{b}}\left(<r_{\mathrm{e}}\right)\right\rangle=\mu_{\mathrm{b}}(0)+6.935$ for $n=4$. However, care should be taken when results from the $r^{1 / 4}$ fitting are compared with those from $r^{1 / n}$ fitting, as the photometric structural parameters are affected systematically. In the case of Abell cluster ellipticals, where we have both Sérsic and de Vaucouleurs law fitted parameters, the propagation of the systmatic error is shown in Fig. 6. It can be seen that the more different $n$ is from 4 for the bulges, the larger are the systematic errors involved in the de Vaucouleurs' fitting. However, there was no noticeable difference for the Kormendy relation $\left(\left\langle\mu_{\mathrm{b}}\left(<r_{\mathrm{e}}\right)\right\rangle-r_{\mathrm{e}}\right)$ between the two cases, as the systematic errors were propagating similarly along both axes (Ravikumar 2004; see also Kelson et al. 2000).

In Fig. 7 we have shown a plot of $\mu_{\mathrm{b}}(0)$ against $\log r_{\mathrm{e}}$. The correlation is not good, but here again we see the various kinds of bulges are divided into two distinct groups, as in Fig. 5. 


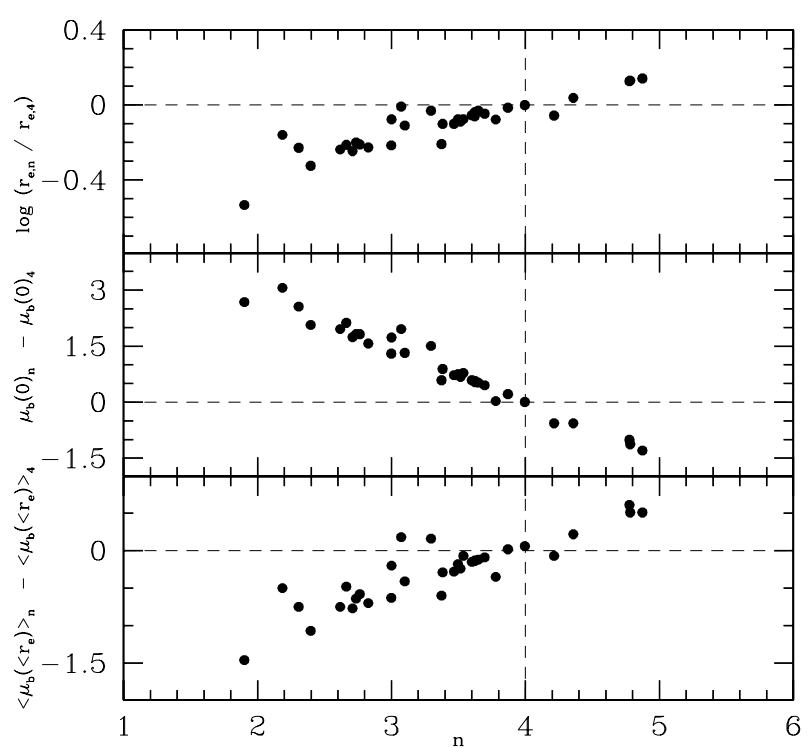

Fig. 6. The systematic error involved when the de Vaucouleurs law is used to extract bulges with a Sérsic profile.

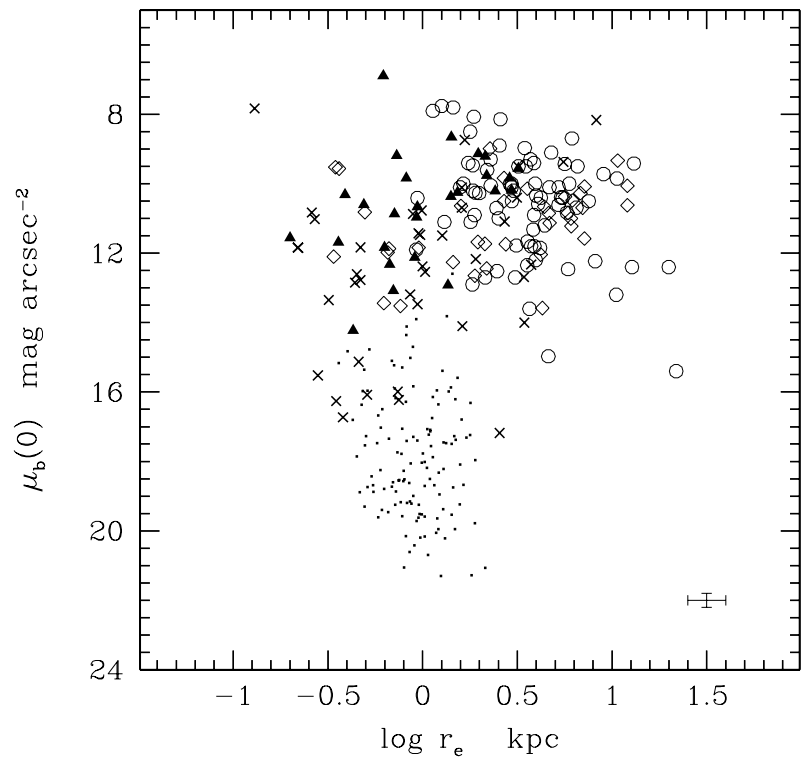

Fig. 7. Variation of $\mu_{\mathrm{b}}(0)$ as a function of effective radius. The symbols are as used in Fig. 4. Typical error bars are also shown.

The lack of correlation seems to go against the Kormendy relation, but the correlation is clearly visible if we plot $\left\langle\mu_{\mathrm{b}}\left(<r_{\mathrm{e}}\right)\right\rangle$ against $\log r_{\mathrm{e}}$, as shown in Fig. 8. When the Sérsic law is used the relation between the two surface brightness terms is a function of $n$, and the lack of a tight $\mu_{\mathrm{b}}(0)-\log r_{\mathrm{e}}$ correlation, is usually attributed to the interdependence of $n$ with these parameters separately (see Khosroshahi et al. 2000b; and Graham \& Guzmán 2003).

It appears at first sight from Fig. 8 that there is large scatter in the Kormendy relation for our bulges, but it is clearly seen that the points are segregated into well correlated subsets. We have given in Table 1 the correlation coefficients and best fit values for the Kormendy relation for the different kinds of bulges. It is seen that the slope for the dEs is significantly different from that for other sets. The errors in the best fit values

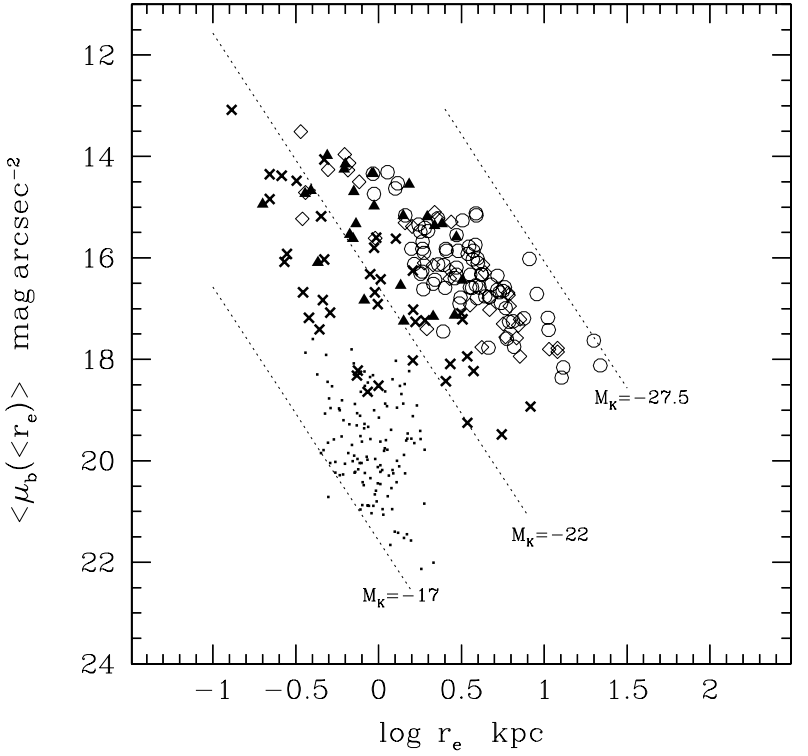

Fig. 8. Kormendy relation for bulges in our sample. The (dashed) lines of constant absolute magnitude are also shown.

Table 1. Kormendy relation, $\left\langle\mu_{\mathrm{b}}\left(<r_{\mathrm{e}}\right)\right\rangle=a \log r_{\mathrm{e}}+b$. The linear correlation coefficient $r$ with significance are also given.

\begin{tabular}{lcccc}
\hline \hline Sample & $a$ & $b$ & $r$ & Significance \\
\hline Es & $1.95 \pm 0.31$ & $15.31 \pm 0.17$ & 0.71 & $>99.9$ \\
S0s & $1.46 \pm 0.49$ & $15.34 \pm 0.19$ & 0.90 & $>99.9$ \\
Early S & $2.60 \pm 0.32$ & $16.86 \pm 0.15$ & 0.48 & 98.7 \\
Late S & $2.53 \pm 0.22$ & $15.14 \pm 0.17$ & 0.74 & $>99.9$ \\
dE & $0.46 \pm 0.38$ & $13.64 \pm 8.14$ & 0.31 & $>99.9$ \\
\hline
\end{tabular}

have been obtained using the bootstrapping and unless otherwise mentioned, we use this re-sampling technique for estimating errors in the rest of the paper.

In Fig. 8 we have shown the line corresponding to $M_{\mathrm{K}}=$ -22 which separates the whole sample into two groups which separately obey a tighter correlation than the combined sample. The slope of the relation for bulges in the brighter group is higher than the slope of the fainter group. Khosroshahi et al. (2004) also reported such a segregation of bulges of early type galaxies in the magnitude space with different slopes for the Kormendy relation. We note that while the S0s and bulges of early type spirals obey the Kormendy relation for the Es in our sample, the bulges of late type spirals form a bridge between the two groups.

Kormendy (1985) also noticed such a "discontinuity" between Es and dEs in the parameter correlations. Interestingly, Fig. 8 shows that the fainter bulges of late type spirals are more similar to the dEs in their slope while their brighter counter parts show a weak similarity with the bulges of E/SOs. Recent studies suggest that bulges of late type spirals are more elongated than those of early type spirals (Fathi \& Peletier 2003). Combining this with the fact that $\mathrm{dEs}$ have greater rotational support than the Es, it is tempting to see the Kormendy relation as an evolutionary diagram but there is too much mixing between the different types, for any clear trend to be discerned (see also Graham \& Guzmań 2003). 

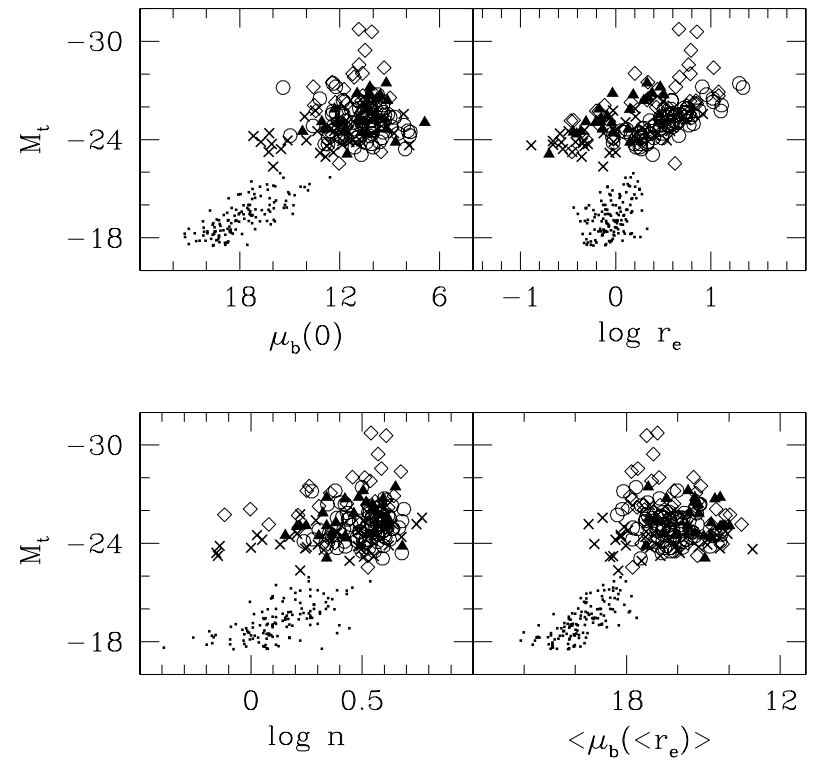

Fig. 9. Correlations of bulge structural parameters $\mu_{\mathrm{b}}(0)$ (in mag $\left.\operatorname{arcsec}^{-2}\right), r_{\mathrm{e}}$ (in kpc), $n$, and $\left\langle\mu_{\mathrm{b}}\left(<r_{\mathrm{e}}\right)\right\rangle$ (in mag $\operatorname{arcsec}^{-2}$ ) with total $K$ magnitude for different galaxies in our sample. For the cluster ellipticals and dEs in our sample, the total luminosity is the same as that of the bulge, as no significant disks were detected in these systems.

\subsection{Correlations with total luminosity}

In Fig. 9 we show the correlations of structural parameters of the bulges in our sample with the total luminosity of the host galaxies. The two surface brightness terms $\left(\mu_{\mathrm{b}}(0)\right.$ and $\left.\left\langle\mu_{\mathrm{b}}\left(<r_{\mathrm{e}}\right)\right\rangle\right)$ seem to correlate well with the total magnitude, even though with large scatter, while for galaxies other than the dEs the half-light radius also shows significant correlation.

\subsection{The photometric plane}

Khosroshahi et al. (2000a) noticed that elliptical galaxies, and the bulges of early type spiral galaxies satisfied a single planar relation of the form $\log n=a \log r_{\mathrm{e}}+b \mu_{\mathrm{b}}(0)+c$. The data points had small scatter around the best fit relation, which Khosroshahi et al. (2000a,b) called the photometric plane (PP). They argued that the tight relation suggested that there is a single formation scenario for the ellipticals and early type bulges. A less direct form of this relation can be seen in Binggeli \& Jerjen (1998) for a sample of dwarf ellipticals and dwarf S0s in the Virgo cluster. For their sample of galaxies containing mostly late type spirals Möllenhoff \& Heidt (2001) noticed a similar relation for the Sérsic parameters obtained by combining observations in near infrared $(J, H$, and $K)$ bands. Khosroshahi et al. (2000a) suggested that the PP can be used in distance determinations, albeit with uncertainties as large as $\sim 50 \%$. Later, Graham (2002) obtained a slightly different form of the PP, from $B$ band observations of early type galaxies in the Virgo and Fornax clusters, and used it to estimate the VirgoFornax distance modulus. A key difference between the photometric plane and the fundamental plane (where the distance estimation error can be $\sim 10 \%$ in rich clusters) is that in the former, all the parameters are photometrically determined, which can be an advantage for large samples of distant galaxies with no spectroscopic data.

In this section we examine the PP relations for the various kinds of bulges of galaxies in our sample. The best fit relations are given in Table 2, where in addition to the coefficients $a, b$, and $c$, we provide the rms scatter along the $\log n$ axis and the angles made by the normal to the PP for each group with that for the Es. An edge-on view of the PP for ellipticals is shown in Fig. 10; these galaxies show a remarkable homogeneity and have significantly smaller rms scatter, about the best fit plane, than the other subsets. The PP coefficients for the bulges of early type spirals and ellipticals are equal, within $1 \sigma$ errors, as shown in Table 2. The PPs for bulges of S0s and late type spirals are, however, significantly different from the PP for ellipticals, as can be seen from the coefficients and the angles between the normals. The scatter about the PP in these cases is also larger than that for the Es. The sample of dwarf galaxies is some what unique in their properties in the sense that they show a PP very similar to that for Es, but with much larger scatter, implying that a high degree of inhomogeneity is present in the individual galaxies.

The PPs for types other than the Es are shown in Fig. 11. Three points are noteworthy here. First, not all bulges follow a common PP. Second, the ellipticals and bulges of early type spirals form a very homogeneous and tight PP while all other samples show a curvature towards the lower end of $n$, in confirmation with recent results (Khosroshahi et al. 2004). Finally, of the two groups of bulges seen in Fig. 3, the bigger and brighter bulges (with larger $n$ values) form a PP with much less scatter than the fainter ones.

The dichotomy is clear in Fig. 12 where we show the histogram of deviations from the PP defined by Es for the combined sample. We have plotted in Fig. 13 the absolute deviation for each galaxy in our sample from the photometric plane for the Es. There is no noticeable trend in the case of $\log n$ and $\log r_{\mathrm{e}}$, possibly because of the significant overlap between the brighter and fainter groups in the distribution of these parameters. However, the $\left|\delta_{\mathrm{PP}}\right|$ show significant correlation with the central intensity and luminosity of the bulges, with the average deviation increasing systematically as these values become fainter.

To examine the distribution of bulges in the 2-dimensional space defined by the photometric plane of the Es, we show a face on view of the PP in Fig. 14. The axes $\boldsymbol{K}_{1}=0.151 \log n+$ $0.989 \log r_{\mathrm{e}}$ and $\boldsymbol{K}_{2}=-0.064 \log n+0.010 \log r_{\mathrm{e}}-0.998 \mu_{\mathrm{b}}(0)$ form orthogonal triad with the normal to the PP for Es. Clearly, the whole available space in the PP is not occupied and, there is suggested distribution of different Hubble types according to their luminosity. There appears to be a (continuous) sequence from the dEs to Es, through the other Hubble types. Elliptical galaxies are considered to be the most relaxed systems, which is consistent with their tight distribution around the FP and PP, and we have seen that the dEs have greater scatter around the PP than other types of galaxies. So the sequence may be tracing a trajectory in the $\boldsymbol{K}_{1} / \boldsymbol{K}_{2}$ plane, which goes from the least to the most relaxed systems. In the next section we try to relate this sequence to the specific entropy of bulges (see Merritt 1999). 
Table 2. The photometric plane coefficients. The number of galaxies in the set $(N)$ and the angle between the normal of the PPs of each set and that of the Es are also given.

\begin{tabular}{lcccrcc}
\hline \hline Sample & $a$ & $b$ & $c$ & $N$ & $\mathrm{rms}_{n}$ & Angle \\
\hline Es & $0.153 \pm 0.022$ & $-0.066 \pm 0.003$ & $1.13 \pm 0.03$ & 76 & 0.037 & - \\
S0s & $0.206 \pm 0.030$ & $-0.106 \pm 0.014$ & $1.54 \pm 0.15$ & 37 & 0.067 & $3.695 \pm 1.620$ \\
Early S & $0.130 \pm 0.040$ & $-0.073 \pm 0.010$ & $1.21 \pm 0.11$ & 26 & 0.058 & $1.354 \pm 1.777$ \\
Late S & $0.232 \pm 0.029$ & $-0.074 \pm 0.006$ & $1.29 \pm 0.07$ & 40 & 0.092 & $4.391 \pm 1.912$ \\
Spirals & $0.213 \pm 0.023$ & $-0.067 \pm 0.005$ & $1.18 \pm 0.06$ & 66 & 0.088 & $3.346 \pm 1.617$ \\
dEs & $0.158 \pm 0.035$ & $-0.082 \pm 0.004$ & $1.60 \pm 0.06$ & 128 & 0.071 & $0.965 \pm 1.202$ \\
\hline
\end{tabular}

Note: $a$ and $b$ are the coefficients of $\log r_{\mathrm{e}}$ and $\mu_{\mathrm{b}}(0)$ respectively, $c$ is the constant and $\mathrm{rms}_{n}$ is the rms scatter in the PP measured along the $\log n$ axis.

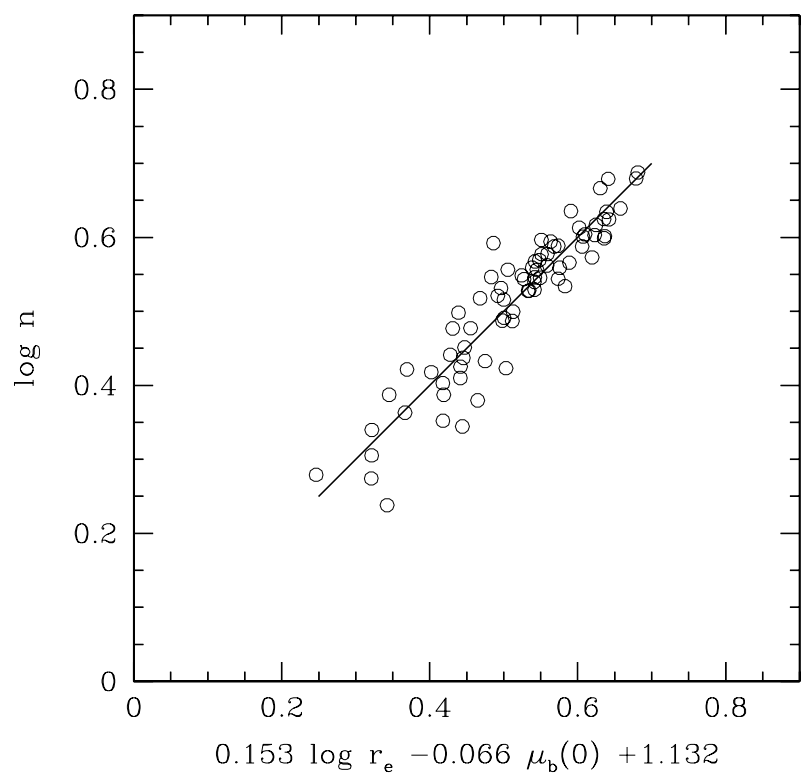

Fig. 10. The photometric plane for Es; an edge-on view. The different sets of cluster ellipticals have a tight distribution about the PP.
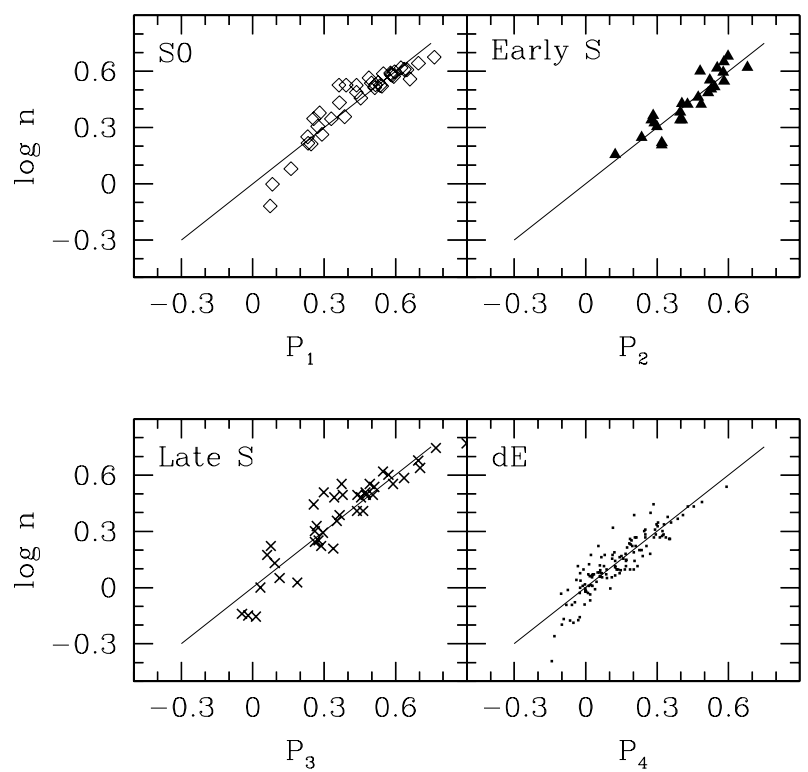

Fig. 11. Edge on view of PPs for S0s (top left window), bulges of early (top right) and late (bottom left) type spirals, and dwarf galaxies (bottom right). $P_{1}=0.21 \log r_{\mathrm{e}}-0.11+1.54, P_{2}=0.13 \log r_{\mathrm{e}}-0.07+$ $1.21, P_{3}=0.23 \log r_{\mathrm{e}}-0.07+1.29$, and $P_{4}=0.16 \log r_{\mathrm{e}}-0.08+1.60$.

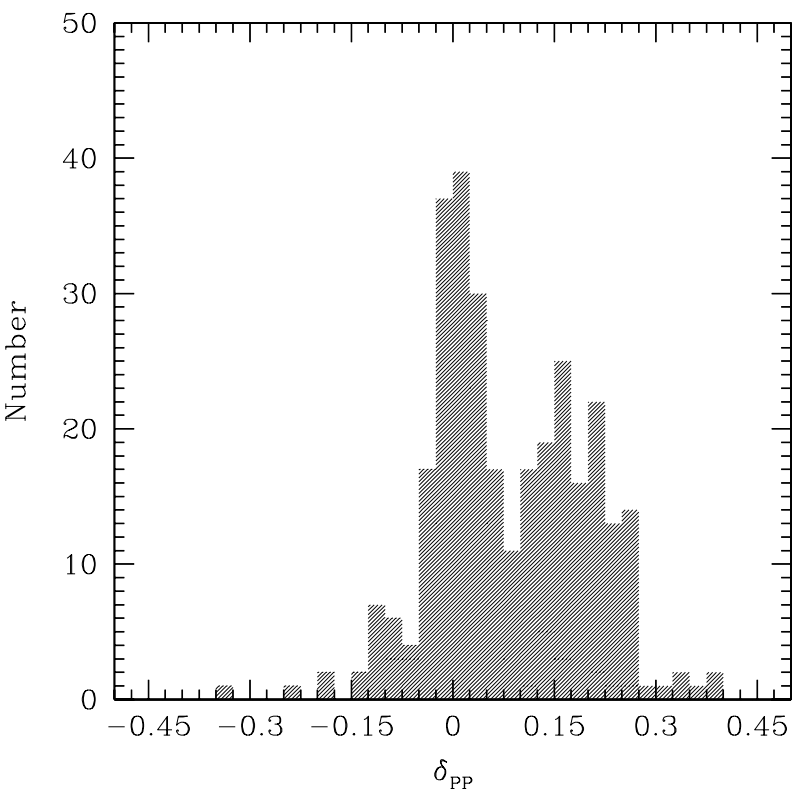

Fig. 12. Histogram of absolute deviations from the PP defined by ellipticals, $\delta_{\mathrm{PP}}=\log n-\left(0.153 \log r_{\mathrm{e}}-0.066 \mu_{\mathrm{b}}(0)+1.132\right)$, for the bulges in the analysis. The presence of two distinct groups is clearly visible.

\section{The specific entropy of bulges}

Galaxies are in a state of constant evolution. However, studies on galaxies of a particular Hubble type show that they indeed display remarkable similarities in their structural properties, manifested by various correlations, photometric and spectroscopic, like the FP and Faber-Jackson relations for the early type galaxies and Tully-Fisher (1977) relation for spirals. The existence of such regularities has interpreted to mean that different classes of galaxies are in a quasi- equilibrium state of their own. Assuming that there exists a local maximum entropy associated with each quasi-equilibrium state, it is possible to estimate the entropy of a self gravitating system, from thermodynamical considerations. Considering the stars in an elliptical galaxy to behave like an isolated, self-gravitating gas, Lima Neto et al. (1999) suggested that the Es might be treated as systems of constant specific entropy. Later, Márquez et al. (2000) showed that the specific entropy of galaxies, estimated from the microscopic Boltzmann-Gibbs definition, increases with merging history. In the latter approach, stars in the galaxies are not required to obey the ideal gas law. Taking their approach 

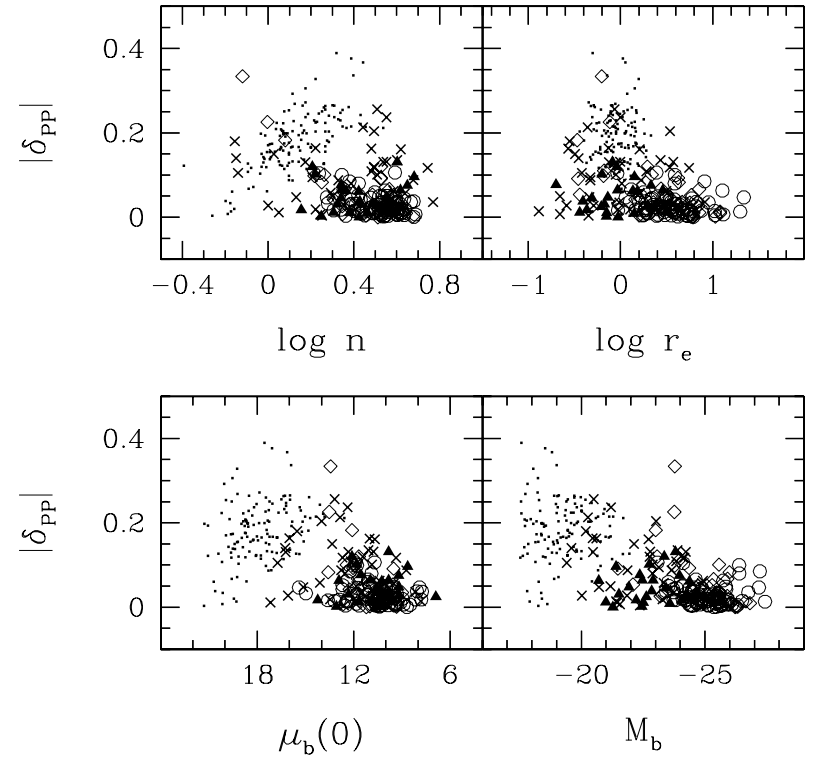

Fig. 13. The variation of $\left|\delta_{\mathrm{PP}}\right|$ as a function of the bulge parameters $n$, $r_{\mathrm{e}}$ (in kpc), $\mu_{\mathrm{b}}(0)$ (in mag $\operatorname{arcsec}^{-2}$ ), and $M_{\mathrm{b}}$ (in $K$ mag). The brighter the central intensity or luminosity of the bulge, the higher the chances that it falls on the PP.

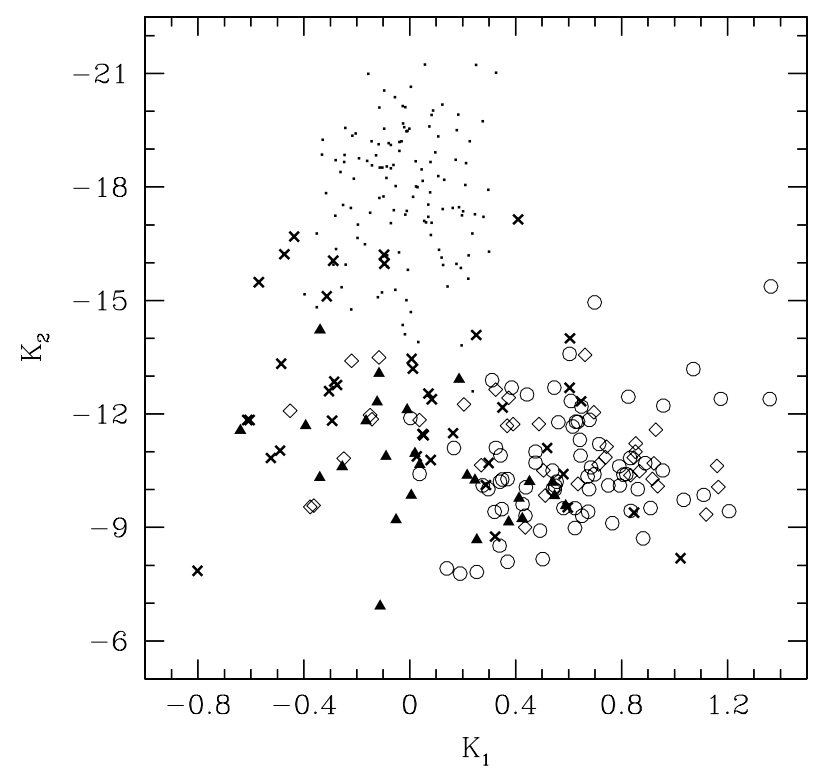

Fig. 14. Distribution of bulges on the PP for Es. The PP for Es is shown face on. The axes are $K_{1}=0.151 \log n+0.989 \log r_{\mathrm{e}}$ and $K_{2}=-0.064 \log n+0.010 \log r_{\mathrm{e}}-0.998 \mu_{\mathrm{b}}(0)$.

further, Márquez et al. (2001) have suggested a theoretical explanation of photometric properties of the Es, including the PP, by constructing an energy-entropy line.

The different evolution scenarios for galaxies can be treated as processes associated with continuous increase in entropy through quasi-stationary states. In the case of hierarchical merging (Kauffmann \& White 1993) or where the violent relaxation mechanism (Lynden-Bell 1967) operates, the phasespace mixing is expected to be fast and hence would result in a higher quantum of change in entropy. On the other hand, in the case of secular evolution (Kormendy 1979) the change
Table 3. Mean specific entropy for bulges in our sample.

\begin{tabular}{lc}
\hline \hline Sample & \multicolumn{1}{c}{$S$} \\
\hline Es & $18.39 \pm 4.44$ \\
SOs & $16.04 \pm 7.35$ \\
Early S & $12.54 \pm 6.22$ \\
Late S & $12.48 \pm 8.32$ \\
Spirals & $12.50 \pm 7.51$ \\
dE & $8.44 \pm 1.98$ \\
\hline
\end{tabular}

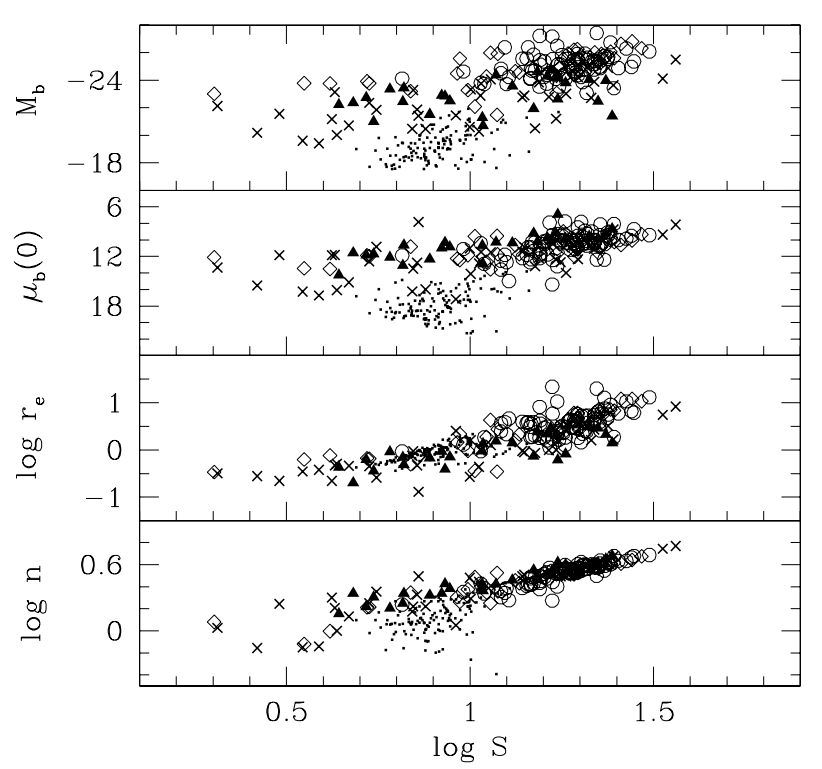

Fig. 15. Variation of $n, r_{\mathrm{e}}$ (in kpc), $\mu_{\mathrm{b}}(0)$ (in mag $\operatorname{arcsec}^{-2}$ ), and $M_{\mathrm{b}}$ (in $K \mathrm{mag}$ ) as a function of specific entropy.

in entropy, one may expect, is rather slow, as the involved time scales are large.

Following the formulation of Márquez et al. (2000; Eq. (2)), the specific entropy of a spheroidal system can be written as,

$S=0.5 \ln I_{\mathrm{b}}(0)+2.5 \ln \frac{r_{\mathrm{e}}}{(1.999 n-0.327)}+F(n)$,

where $F(n)=3.9 n^{-1.3}-1.3 n-0.2 \ln n$.

It should be noted that we have modified the original Márquez et al. (2000) equation by representing the scale radius in kpc (instead of angular units) and neglecting the constant, in order to have a comparison of specific entropy estimates of galaxies in our sample. In Table 3 we show the mean values of specific entropy estimated for the bulges of different Hubble types in our sample. The Es and dEs show a tighter distribution in specific entropy, evident from their dispersion values, suggesting that these structures have higher stability relative to the others. It is clear that the specific entropy decreases systematically as we go from Es to dEs through S0s and spirals. This supports a merging scenario hierarchical or violent relaxation of dEs which then successively climb up in the entropy ladder to form Ellipticals which top the table in specific entropy.

In Fig. 15 we show the variation of specific entropy with the bulge parameters. Clearly there is a systematic increase in the entropy as the bulge becomes brighter and bigger. The distribution of specific entropy of bulges on the photometric plane also shows a systematic pattern as shown in the Fig. 16. As the 


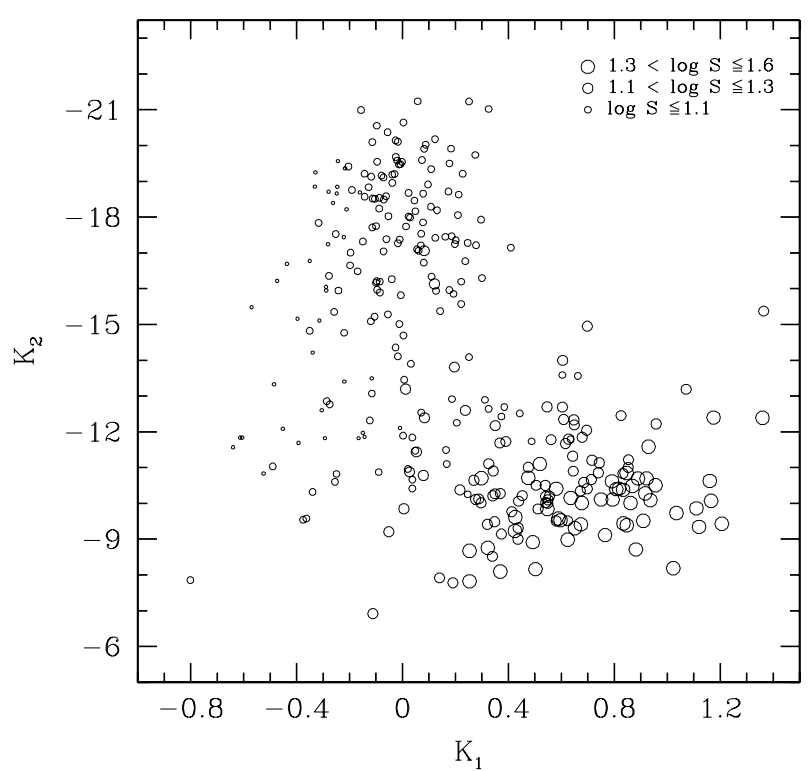

Fig. 16. Specific entropy of bulges and the photometric plane. The axes are as shown in Fig. 14.

luminosity (and hence the mass) of the galaxy is proportional to the central intensity and effective radius squared, the observed trend of specific entropy support a scenario where the less massive systems merge to form more massive ones. The correlation between the Sérsic index and the specific entropy seems to be the tightest, suggesting that the index may reflect the level of interactions the galaxy has passed through.

\section{Discussion and conclusion}

We have compiled from the literature the values of structural parameters for bulges of galaxies of different Hubble types, and have examined distributions of the parameters and two- and three- dimensional correlations between them. The two samples of spheroidal galaxies in our study have been taken from dense environments, while the systems with disks are from the field. However, the differences between cluster and field ellipticals in their photometric properties are marginal if not indistinguishable, with the latter showing an increased (intrinsic) scatter in various correlations (de Carvalho \& Djorgovski 1992; Gebhardt et al. 2003). Hence the inclusion of ellipticals and $\mathrm{dEs}$ from clusters is justified, and the conclusions derived here are valid, even though the homogeneity shown by the Es and dEs separately in our sample may partly have an environmental origin. Comparing the photometric structural parameters of the sample, we find that the distributions of $n, r_{\mathrm{e}}, \mu_{\mathrm{b}}(0)$ and luminosity all show two distinct peaks, with one of the peaks dominated by low luminosity bulges, and the other by high luminosity ones.

The brighter bulges $\left(M_{\mathrm{K}}<-22\right)$ of all Hubble types show similarities in various correlations like the Kormendy diagram and the photometric plane. The fainter bulges show significant differences in their parameter correlations from their brighter counter parts. In the case of the photometric plane, while the brighter bulges show a tight planar distribution, the fainter ones show larger scatter, along with a deviation from the planar distribution for smaller $n$ values. In terms of Hubble types, ellipticals, bulges of early type spirals and lenticulars, especially the brighter ones, form a tight photometric plane, while bulges of late type spirals and faint lenticulars, and dEs, show significant deviations from the plane. Considering this together with the fact that early type (E/SO) bulges are in a relaxed state manifested through their tight fundamental plane relation (Dressler et al. 1987; Djorgovski \& Davis 1987), we suggest here that the tight photometric plane also reflects their relaxed nature.

We have found that the dwarf galaxies show significant similarities in their properties with the faint end bulges of late type spirals. Even though there are contradictory results on the rotational properties of $\mathrm{dEs}$, it is now becoming increasingly evident that in at least a significant fraction of dEs, the oblate structure are supported by rotation (see Pedraz et al. 2002, and the references therein). The bulges of late type spirals are also known to be rotationally supported to a greater extent than the E/S0 galaxies (see for e.g., Proctor \& Sansom 2002), suggesting that bulges of late type spirals could be formed by the merger of dwarfs. This possibility needs to be corroborated by further detailed comparison between bulge and disk properties of spiral galaxies and dEs.

The dichotomy in parameter distributions for bright and faint bulges has been discussed in the literature for various Hubble types. Kormendy (1985) noticed that bright and faint ellipsoids differ significantly in their parameter correlations. van den Bergh (1994) has shown that in lenticular galaxies, fainter bulges are more prolate than the brighter ones, with a non-negligible overlap between the two populations. Bulges of spiral galaxies also show a dichotomy, with later Hubble types being more elongated on an average than their early type counterparts (Fathi \& Peletier 2003). These results are consistent with the differences that we have found, in the parameter distributions and correlations, between the bright and faint bulges. It appears that the difference between the two kinds of bulges is fundamental in nature, and is independent of the Hubble type of the parent galaxy. But in every parameter distribution that we have considered, there is an overlap of bright and faint bulges, making the dividing line rather fuzzy, which helps to account for the contradictory observations on the dichotomy (see Graham \& Guzmán 2003, and the references therein).

Following the prescription of Márquez et al. (2000), we have obtained the specific entropy for the bulges in our sample, using their morphological parameters. The specific entropy for dEs and Es is more tightly distributed than for the other Hubble types, which indicates greater homogeneity for these galaxies and suggests that they are at a local maximum of the entropy, i.e., of stability. The average specific entropy shows a systematic increase as we move towards the earlier types along the Hubble sequence, supporting successive merging of smaller components as in the case of hierarchical clustering (Kauffmann \& White 1993). This observation is in accordance with the results obtained by Márquez et al. (2000), who found that simulated ellipticals showed systematic increase in their specific entropy with successive merging.

For the bulges of lenticulars and late type spirals, we find that the parameter correlations are less tight than those for Es (Figs. 4 and 5). This inhomogeneity is also evident in the case 
of the photometric plane, where their rms scatter is larger than that for Es. Interpreting this as evidence for the existence of different scenarios of formation for the bright and faint bulges helps to understand the observation. It is natural to expect bigger and brighter bulges with large $n$ and $r_{\mathrm{e}}$ values to be the remnants of major mergers. Khosroshahi et al. (2004) observed a strong tendency for $n$ to systematically increase towards the cluster center. Recent studies on luminosity functions also suggest that there is a pronounced dominance of bright end bulges near the central regions of clusters (de Propris et al. 2003) which is usually attributed to the enhanced merging near the centers of clusters occurred while the cluster is forming.

The fainter bulges, irrespective of their Hubble class, might have experienced fewer encounters allowing them to evolve secularly for a longer time in the past (see Kormendy \& Kennicutt 2004, for a recent review). The existence of stable structures like spiral arms, rings and bars are evidence for the lack of any major mergers/interactions in the recent past, as simulations show that major mergers mix up the phase space rather quickly to destroy such fine structures (see Fritze-v. Alvensleben 2004). This idea is corroborated by the observation that the fine structure parameter $\Sigma$ (Schweizer et al. 1990), which characterizes ripples, shells, plumes, boxiness, structures etc. (in field S0s) correlates with deviations in the Color-Magnitude diagram towards the bluer colors (Fritze-v. Alvensleben 2004). This implies that fainter S0s contain a statistically larger number of structures, signifying their lack of strong interactions.

The distribution of spiral galaxies, more abundant in the field and in the outer regions of galaxy clusters, also seems to support the idea that they are likely to have encountered less violent interactions. Studying the properties of disk galaxies as a function of their isolation, Varela et al. (2004) reported that late type spirals (Sc) are more abundant amongst isolated galaxies, while lenticulars are abundant in perturbed systems. Also isolated systems have a remarkable absence of big, bright and massive galaxies, while the opposite is true for perturbed systems. Since the bulge properties of early type spirals show significant similarity with those of bright Es and S0 bulges, one might expect a significant fraction of early type spirals to be closer to denser environments, than their fainter counterparts, so as to be able to experience stronger interactions.

A recent observation significant for this study is that while the fraction of Es in clusters remain almost a constant from $z=0.5$ to $z=0$, that for spirals decreases and that for S0s increases by the same factor of $\sim 5$ (Couch et al. 1998; Fasano et al. 2001). This has been used to suggest that a large fraction of spirals must have transformed to lenticulars in the last $\sim 5$ Gyr. Another important factor about S0s is that a significant fraction of their bulges are as bright as the Es. As higher luminosities are attained only by major mergers, since smaller interactions or secular evolution cannot increase the phase space density by more than a factor of two (see Fritzev. Alvensleben 2004), brighter [field] S0s might be remnants of mergers of (early type) spirals, where the non vanishing or re-distributed angular momentum preserves the presence of a stellar disk. This suggests a strong inter-dependence between morphological evolution and the cosmological large scale structure formation.

Acknowledgements. We thank S. K. Pandey, Yogesh Wadadekar and Somak Raychaudhury for helpful comments and discussions. C.D.R. and S.B. thank IUCAA for the hospitality and the use of facilities there without which this work could not have been done. C.D.R. thanks le Centre Franco-Indien pour la Promotion de la Recherche Avancée (CEFIPRA) for a post-doctoral fellowship during which part of the work presented in the paper has been done.

\section{References}

Anderson, S. 2003, A\&A, 409, 37

Andredakis, Y. C., Peletier, R. F., \& Balcells, M. 1995, MNRAS, 275, 874

Balcells, M., \& Peletier, R. F. 1994, AJ, 107, 135

Barway, S., Mayya, Y. D., Kembhavi, A. K., \& Pandey, S. K. 2005, AJ, 129, 630

Binggeli, B., \& Cameron, L. M. 1993, A\&AS, 98, 297

Binggeli, B., \& Jerjen, H. 1998, A\&A, 333, 17

Bower, R. G., Lucey, J. R., \& Ellis, R. S. 1992, MNRAS, 254, 589

Capaccioli, M., Caon, N., \& D’Onofrio, M. 1992, MNRAS, 259, 323

Couch, W. J., Barger, A. J., Smail, I., et al. 1998, ApJ, 497, 188

Courteau, S. 1996, ApJS, 103, 363

de Carvalho, R. R., \& Djorgovski, S. ApJ, 1992, 389, L49

de Propis, R., et al. 2003, MNRAS, 345, 725

de Vaucouleurs, G. 1959, Hdb. d. Physik, 53, 311

Djorgovski, S., \& Davis, M. 1987, ApJ, 313, 59

D’Onofrio, M., Capaccioli, M., \& Caon, N. 1994, MNRAS, 271, 523

Dressler, A., Lynden-Bell, D., Burstein, D., et al. 1987, ApJ, 313, 42

Faber, S. M., \& Jackson, R. E. 1976, ApJ, 204, 668

Fasano, G., Poggianti, B., Couch, W., et al. 2001, Ap\&SS, 277, 417

Fathi, K., \& Peletier, R. F. 2003, A\&A, 407, 61

Freeman, K. C. 1970, ApJ, 160, 811

Fritze-v. Alvensleben, U. 2004 [arXiv: astro-ph/0407358]

Gerbal, D., Lima Neto, G. B., Marquez, I., \& Verhagen, H. 1997, MNRAS, 285, L41

Gerbhardt, K., Faber, S. M., Koo, D. C., et al. 2003, 597, 239

Graham, A. W., \& Colless, M. 1997, MNRAS, 287, 221

Graham, A. W., \& Ervin, P., \& Caon, N. 2001, AJ, 563, 11

Graham, A. W, \& Guzmán, R. 2003, AJ, 125, 2936

Grosbøl, P. J. 1985, A\&AS, 60, 261

Hinz, J. L., Rieke, G. H., \& Caldwell, N. 2003, AJ, 126, 2622

Hubble E. P. 1936, The Realm of the Nebulae (New Haven: Yale University Press)

Kauffmann, G., \& White, S. D. M. 1993, MNRAS, 261, 921

Kelson, D. D., Illingworth, G. D., van Dokkum, P. G., \& Franx, M. 2000, ApJ, 531, 137

Khosroshahi, H. G., Wadadekar, Y., \& Kembhavi, A. 2000a, ApJ, 533, 162

Khosroshahi, H. G., Wadadekar, Y., Kembhavi, A., \& Mobasher, B. 2000b, ApJ, 531, L103

Khosroshahi, H. G., Raychaudhury, S., Ponman, T. J., et al. 2004, MNRAS, 349, 527

Kormendy, J. 1977, ApJ, 218, 333

Kormendy, J. 1979, ApJ, 227, 714

Kormendy, J. 1985, ApJ, 295, 73

Kormendy, J., \& Kennicutt, R. C. Jr, 2004, ARA\&A, 42, 603

Lima Neto, G. B., Gerbal, D., \& Márquez, I. 1999, MNRAS, 309, 481

Lucey, J. R., Guzmán, R., Carter, D., \& Terlevich, R. J. 1991, 253, 584

Lucey, J. R., Guzmán, R., Steel, J., \& Carter, D. 1997, MNRAS, 287, 899 
Lynden-Bell, D. 1967, MNRAS, 136, 101

Márquez, I., Lima Neto, G. B., Capelato, H., Durret, F., \& Gerbal, D. 2000, A\&A, 353, 873

Márquez, I., Lima Neto, G. B., Capelato, H., Durret, F., Lanzoni, B., \& Gerbal, D. 2001, A\&A, 379, 767

Mathieu, A., Merrifield, M. R., \& Kuijken, K. 2002, MNRAS, 330, 251

Mobasher, B., Guzmán, R., Aragón-Salamanca, A., \& Zepf, S. 1999, MNRAS, 304, 225

Möllenhoff, C., \& Heidt, J. 2001, A\&A, 368, 16

Press, W. H., Teukolsky, S. A., Vetterling, W. T., \& Flannery, B. P. 1992, Numerical Recipes in C 2nd edn. (Cambridge: Cambridge Univ. Press)

Pedraz, S., Gorgas, J., Cardiel, N., Sánchez-Blázquez, P., \& Guzmán, R. 2002, 332, L59

Proctor, R. N., \& Sansom, A. E., MNRAS, 333, 517
Ravikumar, C. D. 2004, Ph.D. Thesis, Photometric and Fundamental Plane Studies of Galaxies, Cochin University, India

Sandage, A., \& Tammann, G. A. 1987, A revised Shapley-Ames Catalog of bright galaxies, Carnegie, Washington (RSA)

Sérsic, J. L. 1968, Atlas de Galaxies Australes, Cordoba, Observatorio Astronomica

Schweizer, F. 1990, ApJ, 364, 335

Trujillo, I., Aguerri, J. A. L., Gutiérrez, C. M., Caon, N., \& Cepa, J. 2002, ApJ, 573, 9

Trujillo, I., Graham, A. W., \& Caon, N. 2001, AJ, 122, 1707

van den Bergh, S. 1994, AJ, 107, 153

Varela, J., Moles, M., Márquez, I., et al. 2004, A\&A, 420, 873

Vazdekis, A., Trujillo, I., \& Yamada, Y. 2004, ApJ, 609L, 458

Tully, R. B., \& Fisher, J. R. 1977, A\&A, 54, 661,

Wadadekar, Y., Robbason, R., \& Kembhavi, A. 1999, AJ, 117, 1219

Young, C. K., \& Currie, M. J. 1994, 268, 11, 